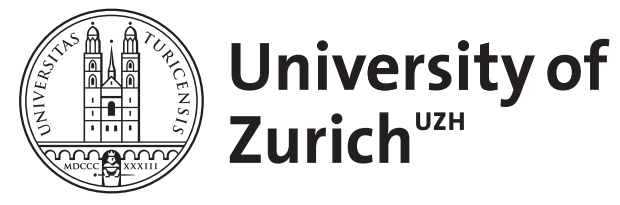

Zurich Open Repository and Archive

University of Zurich

Main Library

Strickhofstrasse 39

CH-8057 Zurich

www.zora.uzh.ch

Year: 2010

\title{
Geld oder Anerkennung? Zur Ökonomik der Auszeichnungen
}

Frey, Bruno S

DOI: https://doi.org/10.1111/j.1468-2516.2009.00319.x

Posted at the Zurich Open Repository and Archive, University of Zurich

ZORA URL: https://doi.org/10.5167/uzh-44409

Journal Article

Accepted Version

Originally published at:

Frey, Bruno S (2010). Geld oder Anerkennung? Zur Ökonomik der Auszeichnungen. Perspektiven der Wirtschaftspolitik, 11(1):1-15.

DOI: https://doi.org/10.1111/j.1468-2516.2009.00319.x 
Thünenvorlesung des Vereins für Socialpolitik

9. September 2009

\title{
Geld oder Anerkennung? \\ Zur Ökonomik der Auszeichnungen
}

\author{
Bruno S. Frey* \\ Universität Zürich \\ und
}

CREMA-Center for Research in Economics, Management and the Arts, Switzerland

Die Thünen-Vorlesung halten zu dürfen ist für mich eine Freude und Auszeichnung wobei wir schon beim Thema wären. Aber was hat Johann Heinreich von Thünen mit Auszeichnungen zu tun? Was zuerst mühsam gesucht erscheint, erweist sich als unmittelbar, wenn die folgende Aussage berücksichtigt wird:

\footnotetext{
„Lang ist die Liste der Ehrungen, die von Thünen zuteil wurden. Ihm persönlich waren die Verleihungen der Ehrenbürgerschaft des Stadt Teterow und die Ehrenmitgliedschaft des Mecklenburgischen Patriotischen Vereins besonders wertvoll und wichtig“ (Bartz und Zühlsdorf 1994: 58).
}

Ehrenbürger einer nicht besonders bedeutenden Stadt oder Ehrenmitglied eines (zumindest heute) belanglosen Vereins zu werden, mag uns wohl als unwichtig erscheinen. Diese Auszeichnungen aber haben Thünen offensichtlich sehr gefreut und in seinem wissenschaftlichen Streben unterstützt. Auf jeden Fall hat er einen bedeutenden Beitrag zur Wirtschaftswissenschaft geleistet: Er hat als erster die Agrarlehre wissenschaftlich betrieben; hat die Agrarökonomie begründet, die Grenzproduktivitätstheorie der Entlohnung abgeleistet und Vorstellungen zu einem

\footnotetext{
* Institut für Empirische Wirtschaftsforschung, Universität Zürich, Winterthurerstrasse 30, CH-8006 Zürich. E-mail: bsfrey@iew.uzh.ch.
} 
gerechten Lohn entwickelt. Seine Beiträge werden in allen ernsthaften

Dogmengeschichten der Ökonomik gewürdigt. Samuelson (1983: 166) nennt Thünen sogar „One of the greatest microeconomists of all time". Nun stellt sich die Frage, ob Thünen seine Auszeichnungen für seine wissenschaftlichen Leistungen erhalten hat oder ob die Auszeichnungen ihn zu seinen wissenschaftlichen Leistungen motiviert haben. Unsere Forschungen sollen dazu beitragen, Antworten auf diese und ähnliche Fragen zu finden.

\section{Wo gibt es Auszeichnungen?}

Häufig werden Auszeichnungen als typisches Merkmal einer monarchisch geprägten Gesellschaft angesehen. Die im Vereinigten Königreich vergebenen Orden wie der Hosenbandorden oder die Ernennung zum Knight oder Lord sind wohl jedem bekannt. Die Vorstellung, dass Auszeichnungen vor allem in Monarchien vergeben werden, ist jedoch verfehlt, wie unsere Untersuchungen auf Grundlage des International Who's Who (Neal, 2006) zeigen. In diesem Werk werden Führungspersönlichkeiten auf der ganzen Welt u.a. nach den von ihnen erhaltenen Preisen befragt. Dieser Ansatz erscheint uns die einzige Möglichkeit zu erfassen, für welche Leistungen und in welchen Bereichen Auszeichnungen vergeben werden ${ }^{1}$. Zahl und Eigenschaften von Auszeichnungen auf der Geberseite zu messen ist nicht praktikabel, weil auf der ganzen Welt Abertausende, wenn nicht Millionen von Institutionen existieren, die Auszeichnungen vergeben. Viele sind überdies nicht bereit, offen zu legen, an wen und wofür sie gegeben wurden.

Tabelle 1 gibt eine Übersicht über die durchschnittliche Zahl von Auszeichnungen in verschiedenen Ländern. Daraus ist unmittelbar ersichtlich, dass die an der Spitze stehenden zehn Länder mit wenigen Ausnahmen (dem Vereinigten Königreich und Spanien) keine Monarchien sind. Einige dieser Länder sind sogar ausgeprägte Republiken, wie etwa Finnland oder die Schweiz. Andere, wie das ganz oben

\footnotetext{
${ }^{1}$ Verzerrungen, die sich aus den Selbstangaben ergeben könnten, sind für die Fragestellung zu verschmerzen, wenn sie sich nicht nach Bereich unterscheiden. Überdies stellt das unterschiedliche Ausmass der Auflistung eine implizite Gewichtung der subjektiven Bedeutung der Auszeichnungen dar: Wichtige Auszeichnungen werden eher angegeben als solche, die von den Empfängern als unwichtig angesehen werden.
} 
stehende Kanada oder Australien, mögen zwar nominell Monarchien sein, verstehen sich aber eindeutig als Republiken.

Tabelle 1 etwa hier

Ein ähnliches Bild ergibt sich, wenn nur vom Heimatstaat verliehene Auszeichnungen (rechte Seite von Tabelle 1) betrachtet werden. Hier finden sich wiederum überwiegend Republiken, insbesondere an zweit oberster Stelle die stolze „République Française“. Daraus lässt sich schliessen, dass Auszeichnungen weniger monarchisch geprägt sind als häufig vermutet wird.

Sind Auszeichnungen eine militärische Angelegenheit? Auch hier sind wir durch Bilder beeinflusst, die Generäle und andere Offiziere mit Orden behangen zeigen. Unsere empirischen Untersuchungen auf Grundlage des International Who's Who verwerfen auch diese Vorstellung.

Umgekehrt könnte vermutet werden, dass im akademischen Bereich, in der ja die reine Suche nach Wissen im Vordergrund stehen sollte, wenige Auszeichnungen verliehen werden. Gemäss Tabelle 2 gilt jedoch eher das Umgekehrte. In den zehn an der Spitze stehenden Ländern werden zwischen 66 Prozent (in der Schweiz und in Belgien) und 43 Prozent aller Auszeichnungen an Personen in der Wissenschaft verliehen. In Deutschland werden etwas mehr als die Hälfte aller Auszeichnungen in diesem Bereich verliehen.

Tabelle 2 etwa hier

Wie auf der rechten Seite von Tabelle 2 ersichtlich ist, stehen dabei die Vereinigten Staaten als heute führende Wissenschaftsnation mit durchschnittlich mehr als 9 Auszeichnungen weit vorne, und auch die Wissenschaftler und Wissenschaftlerinnen in Frankreich, dem Vereinigten Königreich und Kanada erhalten im Schnitt zahlreiche Auszeichnungen. Im akademischen Bereich haben Auszeichnungen sogar einen Siegeszug angetreten. So vergibt zum Beispiel der Verein für Socialpolitik gegenwärtig drei Auszeichnungen:

- den Gossen Preis, - $\quad$ den Gustav-Stolper Preis, und 
- den Reinhard-Selten-Preis.

Auch die Thünen Vorlesung könnte zu diesen Auszeichnungen gerechnet werden. Die European Economic Association vergibt drei Auszeichnungen (den Yrjö Jahnsson Award, den Hicks-Tinbergen Award, und den Young Economists Award, soeben abgelöst durch den FEEM-Award) und hat mehr als 160 Mitglieder zu Fellows ernannt. Ausserdem können die Schumpeter Lecture und die Marshall Lecture als Auszeichnungen betrachtet werden. Die beiden Vereinigungen imitieren damit das Verhalten der grössten Ökonomiegesellschaft, der American Economic Association. Sie zählt auf ihrer Homepage sieben Honors and Awards auf, darunter die John Bates Clark Medal, Distinguished Fellow, Foreign Honorary Member und die Francis A. Walker Medal. Im akademischen Bereich kommen natürlich bereits traditionelle Auszeichnungen wie Ehrendoktorate oder Ehrensenatoren-Titel hinzu. Auch wer den Markt und dessen monetäre Entlohnung als das non plus ultra ansieht, dem gefällt diese Form der Anerkennung offensichtlich. Dazu zählen etwa Milton Friedman oder Gary Becker, die im Economists' Who is Who (Blaug and Vane 2003) 50, respektive 26 Auszeichnungen aufführen.

Sind für die in der Wirtschaft tätige Personen Auszeichnungen weniger von Bedeutung, da ihr wichtigstes Ziel das Geldverdienen sein sollte? Die von uns erhobenen Daten sprechen eine andere Sprache: Gerade in der wirtschaft werden besonders viele Auszeichnungen vergeben. An der Spitze finden sich einige reiche und dynamische Länder wie Kanada, Singapur, die Vereinigten Staaten, Korea und Schweden.

Tabelle 3 etwa hier

Überraschenderweise gehen über 20 Prozent der Auszeichnungen im Königreich Saudi Arabien an Geschäftsleute; in Korea und Singapur 15 Prozent. In den USA, China, Israel und Pakistan sind es immerhin noch 10 Prozent. Privatwirtschaftliche Auszeichnungen wie „Manager des Jahres“, oder „Mitarbeiter des Monats“ spielen somit in der Wirtschaft eine erheblich Rolle.

Auf Auszeichnungen in der Kunst und im Sport braucht hier nicht eingegangen werden. Sie sind offensichtlich wichtig, wie die riesigen Zuschauerzahlen bei der 
Verleihung der „Oscars“ oder bei der Ernennung zum „Sportler (oder zur Sportlerin) des Jahres“ (in den verschiedensten Disziplinen) zeigen.

\section{Motivation und Auszeichnungen}

Die Ökonomik beschäftigt sich hauptsächlich mit materiellen extrinsischen Anreizen in der Form von Geld. Sie tut dies mit Recht, denn Einkommen wird in unserem Wirtschafts- und Gesellschaftssystem als von überragender Bedeutung angesehen. Im Vergleich dazu sind die materiellen Nebeneinkommen (fringe benefits) in Form von Geschäftswagen oder Firmenwohnungen weniger wichtig. Die moderne Forschung in der Psychologischen Oekonomik (oder behavioural economics) weist indessen auf die grosse Bedeutung der intrinsischen Motivation hin. Sie bestimmt das Verhalten nicht nur in all jenen Sektoren, in denen Geldentlohnung nicht im Vordergrund steht (wie etwa im gesamten nicht-gewinnorientierten Bereich), sondern spielt auch bei im Wettbewerb stehenden kapitalistischen Unternehmungen eine grosse Rolle.

Auszeichnungen betreffen eindeutig die extrinsische Motivation. Sie signalisieren Anerkennung für besondere Leistungen nach aussen. Den Empfängern bringen sie Status, was die allermeisten Menschen anstreben, und zuweilen sogar als einziges Ziel ansehen. Sie sind jedoch ihrer Natur nach nicht-materiell, sondern bestehen aus einem Zertifikat, einer Medaille oder sogar nur in einem Händedruck des Vorgesetzten dies aber immer nach aussen sichtbar in Form einer Zeremonie, oder einer Publikation in der Firmenzeitung, auf dem Intra- oder Internet. Manche Auszeichnungen sind zwar mit Geldpreisen verbunden; diese dienen jedoch hauptsächlich dazu, die Ernsthaftigkeit und Bedeutung einer Auszeichnung zu signalisieren und damit zu etablieren. Heute könnte man sich die Nobelpreise gut ohne jegliches Geld vorstellen; sicherlich würden fast alle Wissenschaftler diese Auszeichnungen gerne auch ohne Geld entgegennehmen. Dass Auszeichnungen auch materielle Vorteile bringen, ist allerdings nie vollständig auszuschliessen. Auszeichnungen führen häufig nachträglich zu einer Einkommenserhöhung, indem die besonderen Fähigkeiten der Ausgezeichneten nach aussen kommuniziert werden. Auszeichnungen sind somit häufig mit Geld verbunden, dennoch sollten Auszeichnungen nicht einfach als ein geldähnliches Phänomen angesehen werden. Vielmehr gibt es verschiedene grundsätzliche Unterschiede, die es herauszuarbeiten lohnt. 


\section{Unterschiede zwischen Auszeichnungen und Geld}

Auszeichnungen unterscheiden sich in mehrfacher Hinsicht deutlich von

Geldleistungen:

- Asymmetrie: Eine Auszeichnung ist für den Geber oft beinahe kostenlos, kann aber den Nutzen des Empfängers gewaltig steigern. Bei monetären Leistungen hingegen entsprechen sich die beiden Seiten (abgesehen vom abnehmenden Grenznutzen des Geldes).

- Beziehungen: Mit dem Empfang einer Auszeichnung wird eine soziale Beziehung etabliert. Der Empfänger geht eine gewisse Loyalitätsverpflichtung ein, ansonsten hätte er auf die Ehrung verzichten müssen. Wer gar nicht die Möglichkeit hat eine Auszeichnung abzulehnen, wird sich tendenziell den Zielen des Gebers annähern um die kognitive Dissonanz zu verringern. Bei Geldzahlungen entsteht hingegen keine Loyalitätsverpflichtung: wer eine Leistung gegen Geld erstellt, kann sich auf den materiellen Vorteil berufen und braucht mit den Zielen des Zahlers nicht überein zu stimmen.

- Leistung: Auszeichnungen werden in der Regel für eine allgemeine, oft sogar vage Leistung wie etwa dem „Lebenswerk“ verliehen. Da Geldzahlungen notwendigerweise exakte Summen sind, muss auch die Leistung entsprechend messbar und kommunizierbar sein. Dies äussert sich am deutlichsten in der aus der Prinzipal-Agenten Theorie stammenden Forderung nach „Leistungsentlohnung“: das für die Arbeitsleistung bezahlte Gehalt sollte möglichst eng mit der (irgendwie definierten) Leistung verknüpft sein. Eine einmalige Geldzahlung für ein Lebenswerk lässt sich jedoch schwer vorstellen, denn eine „Lebensleistung“ lässt sich nicht vernünftig in Geldeinheiten messen. Dafür ist eine Auszeichnung wesentlich besser geeignet, denn damit kann eine allgemeine Wertschätzung ausgedrückt werden.

- Verleihung: Auszeichnungen werden immer öffentlich in einer Zeremonie verliehen und überdies in Zeitungen oder auf dem Intra- oder Internet veröffentlicht. Die ,gläserne Lohntüte“ ist dagegen weitgehend unbekannt. Die allermeisten Gehälter werden als Privatangelegenheit angesehen. 
- Besteuerung: Wer eine Auszeichnungen erhält, wird nicht zusätzlich besteuert. Geldeinkommen unterliegt hingegen (üblicherweise) einer zuweilen hohen marginalen Besteuerung.

\section{Literatur zu Auszeichnungen}

In der Wirtschaftswissenschaft wurden bisher nicht-materielle Anreize in Form von Auszeichnungen fast völlig vernachlässigt. Ein vor 37 Jahren veröffentlichter Aufsatz (Hansen und Weisbrod 1972) beschäftigt sich ausschliesslich mit Auszeichnungen innerhalb der Volkswirtschaftslehre. Andere Wissenschaften wie die Geschichte konzentrieren sich auf einzelne Orden oder Medaillen (z.B. Risk 1972). Die Soziologie nimmt das Phänomen zwar durchaus zur Kenntnis, bleibt aber häufig im Allgemeinen und unternimmt kaum vergleichende Analysen (z.B. Bourdieu 1979, Braudy 1986, De Botton 2004).

In der Psychologischen Ökonomik, die die wirtschaftswissenschaftlichen Methoden mit Einsichten aus der Psychologie bereichert, vermögen mehrer Ansätze spezielle Aspekte von Auszeichnungen zu beleuchten. Besonders bedeutsam sind Beiträge über Ansehen, Identität, Status und Reputation, Belohnungen, soziale Anerkennung, Reziprozität, Identität, Konventionen und Gebräuche und Superstars und positionale Güter ${ }^{2}$.

Die im Folgenden dargestellte Forschung beruht auf gemeinsamen Forschungen mit Susanne Neckermann und später auch mit Reto Cueni an der Universität Zürich. Einzelne Aspekte wurden bereits veröffentlicht (Frey 2006, 2007, Frey und Neckermann 2006, 2008, 2009) oder sind als Arbeitspapiere erhältlich (www.bsfrey.ch, www.iew.uzh.ch, www.CREMA-research.ch). Ein erheblicher Teil unserer Forschung ist erst im Entstehen.

\section{Fragestellungen und methodische Ansätze}

${ }^{2}$ Vgl. dazu zum Beispiel Akerlof und Kranton 2005, Auriol und Renault 2001, Brennan und Pettit 2004, Dubey und Geanakoplos 2005, Ederer und Patacconi 2004, English 2005, Fehr und Gächter 2000, Fehr und Schmidt 2004, Frank 1985, Hirsch 1976, Loch et al 2001, Rosen 1981, Sururov und van de Ven 2006), Young 1993. 
Auszeichnungen können mannigfaltige Formen aufweisen und die Auswirkungen auf die Empfänger können sich stark unterscheiden. Gleichzeitig gibt es nur eine sehr begrenzte Zahl von empirischen Untersuchungen. Wie bereits erwähnt, sind uns keine Studien bekannt, die systematisch untersuchen, welche Personen für welche Leistungen welche Auszeichnungen erhalten und welche Wirkungen auf die Menge und Qualität der Leistung des Empfängers folgen. Deshalb ist es angezeigt, das Thema von verschiedenen Blickrichtungen her zu betrachten.

Um einen ersten Überblick zur Bedeutung von Auszeichnungen in verschiedenen Ländern und Bereichen zu gewinnen, eignen sich -wie bereits erläutert - Daten über Personen, wie sie im International Who's Who (Neal, 2006) aufgeführt sind. Für einzelne Berufe können spezialisierte Verzeichnisse verwendet werden, so etwa für die Wirtschaftswissenschaften das Who's Who in Economics (Blaug und Vane 2003). Die unterschiedliche Bedeutung von Auszeichnungen lassen sich damit nur annähernd erfassen. Eine Vollerhebung aller Personen verbietet sich aus Gründen des riesigen Aufwands. Aber selbst bei Stichproben (wie wir vorgegangen sind) müsste jede einzelne Auszeichnung, die von irgendeiner der ausgewählten Personen angegeben wurde, mit Listen verglichen werden, aus denen deren Bedeutung entnommen werden kann. Solche Listen existieren jedoch in der Regel nur für ausgewählte Orden. Selbst dann braucht die subjektiv empfundene Bedeutung nicht der formellen Einstufung zu entsprechen. So berichtet mir zum Beispiel ein englischer Kollege, dass er lieber mit einem „Knight Bachelor“ und dem Titel „Sir“ ausgezeichnet würde als zum höher gestellten „Lord“ ernannt zu werden. Ersteres wird eher für wissenschaftliche Leistungen vergeben, während letzteres häufig dazu dient, das Oberhaus mit Anhängern einer bestimmten Partei zu besetzen.

Ein anderes Forschungsziel kann sein, die Bedeutung von Auszeichnungen aus Sicht der Geber zu untersuchen. Aus ökonomischer Sicht ist besonders wichtig, wie die Vorgesetzten in Unternehmungen die Vergabe von Auszeichnungen beurteilen. Dazu eignen sich Interviews mit Managern, die Erfahrungen im Umgang mit Firmenauszeichnungen haben. Wir haben neun Personalverantwortliche aus unterschiedlichen Firmen in strukturierten Interviews gefragt, warum sie Auszeichnungen anstelle von, oder in Kombination mit andern Anreizinstrumenten verwenden. Im Gegensatz zu den Vorstellungen der Standardökonomie sind sich die befragten Manager einig, dass Auszeichnungen die Leistungen der Mitarbeitenden 
über das Niveau anheben können, das mit unmittelbaren und zukünftigen materiellen Anreizen erreichbar wäre. Die Personalleiter sehen Auszeichnungen als zusätzliches Anreizinstrument neben der Leistungsentlohnung und Beförderung. Auszeichnungen sind jedoch in einer Hinsicht einzigartig: sie sind formelle Belohnungen, die aber typischerweise für den Gewinner eine Überraschung darstellen und emotionale Reaktionen hervorrufen, die zu einer engeren Beziehung zur Firma führen. Manager setzen Auszeichnungen vor allem dort ein, wo Leistungsentlohnung versagt oder sogar kontraproduktiv ist (wie etwa bei multi-tasking Situationen).

Die Bedeutung einer Auszeichnung für die Empfänger hängt wesentlich von der Stellung des Gebers ab. Je prominenter und je höher geachtet diese Institution ist, desto höher ist der Nutzen für die Ausgezeichneten. Eine Institution wird aus eigenem Interesse darauf achten, die Zahl der Auszeichnungen knapp zu halten. Dazu dient nicht nur die Beschränkung der absoluten Zahl, sondern es wird auch versucht, möglichst unterschiedliche Auszeichnungen zu schaffen. So kennen zum Beispiel viele Firmen nicht nur eine Auszeichnung für ihre Mitarbeitenden, sondern es gibt sie in Silber, Gold, Platin, mit oder ohne Trophäe etc.

In einigen Fällen zieht der Geber selbst den hauptsächlichen Nutzengewinn aus der Verleihen einer Auszeichnung: Die Auszeichnenden schmücken sich mit dem Prestige der Ausgezeichneten. Dies ist etwa der Fall, wenn eine Universität einem Nobelpreisträger einen Ehrendoktor verleiht, oder Roger Federer zum „Schweizerischen Sportler des Jahres“ gekürt wird.

Im Rahmen der Unternehmensführung, oder allgemeiner der Prinzipal-Agenten Beziehung, stehen die vermuteten und tatsächlichen Anreizwirkungen von Auszeichnungen im Vordergrund. Um diese zu erforschen, eignen sich Vignettenstudien. Sie erlauben es, innerhalb eines virtuellen Experiments die Auswirkungen unterschiedlicher Bedingungen zu erfassen. Wir haben eine online Befragung mit den am IBM Research Labor in Rüschlikon (Schweiz) Beschäftigten durchgeführt (Neckermann und Frey 2007).

Das Experiment zeigt, dass die Befragten systematisch auf die Ankündigung einer Auszeichnung reagieren: Die Bereitschaft zu einem öffentlichen Gut beizutragen steigt monoton mit dem Wert des monetären Bonus oder Geschenks, das die Auszeichnung begleitet. Wir finden somit, dass Gewinner ihren Beitrag zum 
öffentlichen Gut erhöhen. Diese Reaktion ist erklärungsbedürftig. Warum sollte eine Person ihren Beitrag nach dem Erhalt einer Auszeichnung steigern? Die Belohnung ist ja bereits erfolgt und sollte deshalb den darauf folgende Arbeitseinsatz nicht beeinflussen. Den Grund für die nachherige Leistungssteigerung sehen wir in dem Bestreben der Ausgezeichneten, nach aussen hin die Belohnung zu rechtfertigen. Ausserdem identifizieren sie sich mehr mit der Firma und leisten entsprechend bessere Arbeit.

Der Beitrag zum öffentlichen Gut ist signifikant höher, wenn die Gewinner einer Auszeichnung auf dem Intranetz veröffentlicht werden oder wenn sie in einer Zeremonie mit ihren Kollegen gefeiert werden. Diese Öffentlichkeit ist wichtig. Damit wird der Beitrag zum öffentlichen Gut in gleichem Masse erhöht wie eine Steigerung der mit der Auszeichnung einhergehenden Geldsumme von Null auf 2000 Schweizer Franken. Dieser grosse Effekt der Publizität lässt sich auf die Wirkung der gewonnenen Reputation zurückführen, erklärt aber wohl nicht das gesamte Ausmass. Es ist plausibler, wenigstens einen Teil des grossen Effektes auf die soziale Anerkennung zurück zu führen.

Insgesamt folgt aus der Vignettenstudie, dass Auszeichnungen einen signifikanten Einfluss auf die Motivation der Mitarbeitenden ausüben und ihr Verhalten systematisch beeinflusst. Allerdings darf nicht übersehen werden, dass es nicht um tatsächliches, sondern um geäusserte Verhaltensabsichten geht. Ob sich die Mitarbeitenden auch tatsächlich in dieser Weise verhielten, wenn eine neue Auszeichnung eingeführt würde, muss weiter untersucht werden. Andererseits sollte man auch nicht allzu kritisch sein, baut doch auch die Nutzen-Kosten Analyse auf der Methode der geäusserten Präferenzen (stated preference approach) auf.

Beschränkt lassen sich auch Laborexperimente durchführen. Ein wohlbekannter Vorteil besteht in der Möglichkeit, die Wirkungen präzise auf die Vergabe von Anreizen zurück zu führen und andere Einflüsse auszuschalten. Neben dem allgemeinen Problem der externen Validität des Experiments besteht das Hauptproblem in den spezifischen Eigenschaften von Auszeichnungen. Sollen sie für unsere Fragestellung relevant sein, darf nicht einfach nur eine Belohnung angeboten und deren Wirkung untersucht werden. Eine Auszeichnung hat eine ausgeprägte soziale Dimension: eine Auszeichnung ist nicht zuletzt soviel wert, wie sie von der 
relevanten Umwelt eingeschätzt wird. Eine dieser Anforderung entsprechende Versuchsanordnung ist nicht einfach zu finden.

Die im Labor auftretende Schwierigkeit, eine glaubwürdige Auszeichnung zu schaffen, lassen sich zumindest teilweise sich durch Feldexperimente überwinden. Neckermann und Kosfeld (2009) zeigen, dass in einer realen Arbeitssituation Auszeichnungen einen signifikant positiven Anreizeffekt auf die Arbeitsleistung haben.

Studien, in denen bereits existierende Daten für die Beziehung zwischen Auszeichnungen und Arbeitsanreizen verwendet werden, sind mit einem grundsätzlichen Problem konfrontiert. Auszeichnungen werden ja besonders erfolgreichen Personen gegeben, von denen angenommen werden kann, dass sie auch in der Zukunft erfolgreicher als andere Personen sein werden. Die Kausalität ist deshalb ungeklärt: führt eine Auszeichnung zu mehr Leistung, oder erhalten fähigere Leute eher Auszeichnungen? In der Regel trifft beides zu. Im Rahmen der PrinzipalAgenten Problematik steht jedoch die Anreizwirkung im Vordergrund. Es gibt sicherlich Möglichkeiten, das Kausalitätsproblem zu lösen. Dazu ist aber ein hohes Mass an Originalität auf Seiten der Forschenden notwendig. Vielleicht lässt sich einmal ein natürliches Experiment finden, in dem Auszeichnungen völlig unabhängig von einer zukünftigen Leistungserwartung vergeben wurden. Mit komplizierten ökonometrischen Techniken kann ebenfalls versucht werden, den kausalen Effekt von Auszeichnungen auf die Arbeitsleistung zu isolieren. Eine von uns durchgeführte Studie (Neckermann, Cueni und Frey 2009) in einer einzelnen Firma wird im nächsten Abschnitt skizziert. Folgende Hypothese soll empirisch getestet werden: Gewinner einer Auszeichnung steigern nach deren Erhalt ihre Leistung.

Zur Untersuchung wird eine Schweizer Unternehmung im Finanzdienstleistungsbereich betrachtet, die uns ungewöhnlich detaillierte Paneldaten über den Arbeitseinsatz ihrer Mitarbeitenden zur Verfügung stellte. Die Leistung der Mitarbeiter wird in mehreren Dimensionen erfasst und in einem Index zusammengefasst. Das Bewertungssystem beurteilt nicht nur den Arbeitseinsatz, sondern auch die Qualität der erbrachten Leistung aus Sicht der Kunden. Die Firma vergibt verschiedene Auszeichnungen. Wir betrachten die zweitunterste, die als „Goldauszeichnung“ bezeichnet ist. Der Gewinner oder die Gewinnerin erhält im 
Rahmen im Beisein der Kollegen eine Urkunde, einen symbolischen Bonus und wird von einem höheren Vorgesetzten beglückwünscht. Diese Auszeichnung wird für besondere Anstrengungen verliehen, die ausserhalb der normalen Tätigkeit der Mitarbeitenden liegen. Damit wird das oben erwähnte Kausalitätsproblem vermieden, denn die Gewinner erhalten ihre Auszeichnung nicht wegen ihrer normalen Leistung, aber wir untersuchen die Auswirkung auf eben diese Leistung. Vor der Verleihung der Goldauszeichnung lässt sich kein systematischer Unterschied zwischen geehrten und nicht geehrten Mitarbeitern feststellen; sie verrichten ihre normale Arbeit in ähnlich guter Weise. Nach Erhalt der Goldauszeichnung erhöht sich die Leistung der ausgezeichneten Personen im nachfolgenden Monat in statistisch signifikanter Weise. Im Folgemonat ist deren Leistung um 7,5 Prozent höher als bei den Kollegen, die keine Auszeichnung erhalten haben. Diese Differenz rührt nicht etwa daher, dass die Nichtgeehrten weniger arbeiten würden, sondern auf eine höhere Leistung der Ausgezeichneten. Besonders hoch ist die Leistungssteigerung bei Personen, die vorher unterdurchschnittlich eingestuft wurden. Es liegt deshalb nahe, die beobachtete Erhöhung der Leistung der Ausgezeichneten auf die Wirkung des sozialen oder psychischen Drucks zurück zu führen: Ein Ausgezeichneter möchte bewusst oder unbewusst den andern Mitarbeitenden zeigen, dass er oder sie zu Recht belohnt wurde.

Die Ergebnisse unserer Studie sind aus mehreren Gründen bedeutsam. Ökonomischer Standardtheorie folgend würde kein Effekt nach Erhalt der Auszeichnung zu erwarten sein, denn für diese Leistungssteigerung wird man ja nicht belohnt. Erstaunlich ist auch, dass die Goldauszeichnung leistungssteigernd wirkt, obwohl die monetären Anreize gering sind.

Unsere Resultate deuten darauf hin, dass Auszeichnungen im Rahmen von PrinzipalAgenten Beziehungen wirksame Anreizinstrumente darstellen. Allerdings ist vor vorschnellen Verallgemeinerungen zu warnen.

\section{Induzierte Veränderungen der Nutzenfunktion}

Der Einsatz von Instrumenten zur Leistungssteigerung kann die Nutzenfunktion der Betroffenen ändern. Die Bedingungen, unter denen monetäre Belohnungen oder Bestrafungen die intrinsische Motivation verstärken (crowding in) oder verdrängen (crowding out) sind wohlbekannt und empirisch gut gesichert (Frey 1992, 1997, Frey 
und Jegen 2001, Bénabou und Tirole 2003). In Abbildung 1 wird diese Beziehung durch den Pfeil (1) veranschaulicht.

\section{Abbildung 1 ungefähr hier}

Beziehungen zwischen anderen Anreizsystemen, wie Auszeichnungen und Motivation wurden hingegen bisher nicht untersucht. Die folgenden Überlegungen sind deshalb spekulativ.

Geldzuwendungen (Pfeil (2) in Abbildung1) haben zwei gänzlich unterschiedliche Wirkungen auf den (Grenz-)Nutzen, den ein Ausgezeichneter erfährt. Ist eine Auszeichnung von einer Geldzahlung begleitet, wird sie als wichtiger und ernsthafter betrachtet, was sie für den Empfänger wertvoller macht. Zahlt jedoch der Empfänger für die Auszeichnung, wird dies als Bestechung angesehen und sie verliert schlagartig an Wert. Würde zum Beispiel publik, dass ein Nobelpreis mit Hilfe von Geldzahlungen erworben wurde, wird die Auszeichnung wertlos und die betreffende Person verliert die Anerkennung sowohl seiner Kollegen als auch von Aussenstehenden.

Auszeichnungen können das Einkommen steigern, wenn bisher verborgene Fähigkeiten nach aussen signalisiert werden. Dies ist als Pfeil (3) in Abbildung 1 wiedergegeben. Dieses Signal könnte etwa dazu führen, dass Personen, die in einer Firma ausgezeichnet werden, mit einem höheren Gehalt abgeworben werden. Ausserhalb der Firmen führt eine Auszeichnung zu mehr Beachtung und Prominenz, was sich etwa in höheren Salären für Vorträge oder lukrativeren Buchprojekten äussert (Malmendier und Tate 2008).

Von Auszeichnungen ist auch eine direkte Wirkung auf die intrinsische Motivation zu erwarten. (Pfeil (4) in Abbildung 1). Auszeichnungen wirken somit unterstützend, was die intrinsische Motivation verstärkt. Ein wesentliches Kennzeichen von Auszeichnungen ist ja das öffentlich ausgesprochene Lob für die erbrachte Leistung. Damit ist eine wesentliche Bedingung für eine Steigerung der intrinsischen Motivation erfüllt. Empirische Untersuchungen können diese Hypothesen natürlich verwerfen, was ich allerdings für wenig wahrscheinlich halte.

\section{Ausbreitungseffekte}


Auszeichnungen können den Nutzen verschiedener Personen beeinflussen. Im Zentrum steht die Wirkung auf die Geehrten. Falls der Personenkreis, in dem die Auszeichnung beachtet wird, einem Nullsummenspiel verhaftet ist, fühlen sich die Nicht-Ausgezeichneten als Verlierer, was ihre Leistungsmotivation senken kann. Wie erwähnt, hat allerdings unsere empirische Untersuchung für Beschäftigte in einer Finanzdienstleistungsfirma gezeigt, dass kein negativer Effekt auftritt. Auch wer nicht gewinnt, kann eine Chance sehen, in der Zukunft ausgezeichnet zu werden und wird deshalb seine Leistung nicht verringern. Eine Auszeichnung kann schliesslich auch die Allgemeinheit beeinflussen. Sie kann als expressiver Akt oder als Signal dafür angesehen werden, was die vergebende Institution als wertvolles Handeln ansieht. Wer etwa von der englischen oder holländischen Königin am Ende seiner Karriere zum Ritter ernannt wird, erreicht eine Vorbildfunktion für andere Personen, die ihm oder ihr nachahmen sollen. Gerade bei Orden und Medaillen dürfte dieser dritte Effekt besonders wichtig sein - aber besonders schwer zu untersuchen.

\section{Abschliessende Bemerkungen}

Die Ausführungen haben deutlich gemacht, dass Auszeichnungen in allen Gesellschaften, und insbesondere auch in der Wirtschaft, wichtig sind. Dennoch sind die Bestimmungsgründe und Auswirkungen von Auszeichnungen in der Wirtschaftswissenschaft noch kaum untersucht worden. Wegen der grossen Zahl unbeantworteter Fragen eröffnet sich ein Gebiet, das fruchtbare Forschungen erlaubt. Noch ist es verfrüh, wirtschaftspolitische Folgerungen zu ziehen, also etwa die „gesellschaftlich optimale“ Zahl von Auszeichnungen zu bestimmen. Immerhin lässt sich schon jetzt zur Kenntnis nehmen, dass gerade besonders dynamische Unternehmungen und Institutionen grossen Wert auf Auszeichnungen legen und davon eine Fülle verleihen. Die Zeit ist für die Ökonomik gekommen, sich von einer alleinigen Konzentration auf monetäre Anreize zu lösen und neben der intrinsischen Motivation auch die motivationale Kraft von anderen nicht-materiellen Auszeichnungen zu beachten.

\section{Literatur}


Akerlof, George A. und Rachel E. Kranton (2005). „Identity and the economics of organizations“. Journal of Economic Perspectives 19(1): 9-32.

Auriol, Emmanuelle und Régis Renault (2001). „Incentive Hierarchies“. Annales d'Economie et de Statistique 63-64: 261-282.

Bartz, Rolf P. und Petral Zühlsdorf (1994). Das Thünen-Museum-Tellow. Auf den Spuren des Johann Heinrich von Thünen. Dülmen: Laumann Druck.

Bénabou, Roland und Jean Tirole (2003). „Intrinsic and Extrinsic Motivation“. Review of Economic Studies 70(3): 489-520.

Blaug, Mark und Howard R. Vane (eds.) (2003). Who's Who in Economics Vol. 4. Cheltenham, UK: Edward Elgar Publishing Ltd.

Bourdieu, Pierre (1979). La Distinction. Critique sociale du jugement. Paris: Les editions de minuit.

Braudy, Leo (1986). The Frenzy of Renown: Fame and ist History. New York: Oxford University Press.

Brennan, Geoffrey und Philip Pettit (2004). The Economy of Esteem: an Essay on Civil and Political Society. Oxford, New York: Oxford University Press.

De Botton, Alain (2004). Status anxiety. New York: Pantheon Books.

Dubey, Pradeep und John Geanakoplos (2005). „Grading in games of status: marking exams and setting wages“.Manuscript Number 1537, Yale University.

Ederer, Florian und Andrea Patacconi (2004). „Interpersonal Comparisons, Status and Ambition in Organisations“. Mimeo, Department of Economics, University of Oxford. 
English, James F. (2005). The economy of prestige: prizes, awards, and the circulation of cultural value. Cambridge, Mass.: Harvard University Press.

Fehr, Ernst und Simon Gächter (2000). „Fairness and Retaliation: The Economics of Reciprocity“. Journal of Economic Perspectives 14(3): 159-181.

Fehr, Ernst und Klaus M. Schmidt (2004). „Fairness and Incentives in a Multi-task Principal-Agent Model“. Scandinavian Journal of Economics 106(3): 453-474.

Fershtman, Chaim, Hans K. Hvide und Yoram Weiss (2001). „Stauts Concerns and the Organization of Work“. Mimeo, Eitan Berglas School of Economics, Tel-Aviv University.

Frank, Robert H. (1985). Choosing the Right Pond: Human Behavior and the Quest for Status. New York: Oxford University Press.

Frey, Bruno S. (1992). „,Tertium Datur: Pricing, Regulating and Intrinsic Motivation“. Kyklos 45(2): 161-184.

Frey, Bruno S. (1997). Markt und Motivation. Wie ökonomische Anreize die (Arbeits-) Moral verdrängen. München: Vahlen.

Frey, Bruno S. (2006). „Giving and Receiving Awards“. Perspectives on Psychological Science 1: 377-388.

Frey, Bruno S. (2007). „Awards as Compensation“. European Management Review 4: 6-14.

Frey, Bruno S. und Reto Jegen (2001). „Motivation Crowding Theory“. Journal of Economic Surveys 15(5): 589-611.

Frey, Bruno S. und Susanne Neckermann (2006). „Auszeichnungen: ein vernachlässigter Anreiz“. Perspektiven der Wirtschaftspolitik 7(2): 271-284. 
Frey, Bruno S. und Susanne Neckermann (2008). „Awards: A View From Psychological Economics“. Journal of Psychology 216 Special Issue: 198-208.

Hansen, J. Lee und Burton Weisbrod (1972). „,Toward a General Theory of Awards, or, Do Economists Need a Hall of Fame?“. The Journal of Political Economy 80(2): $422-431$.

Hirsch, Fred (1976). Social limits to growth. Cambridge, Mass.: Harvard University Press.

Loch, Christoph H., Michael Yaziji und Christian Langen (2001). „The fight fort he alpha position: Channeling status competition in organizations“. European Management Journal 19(1): 16-25.

Malmendier, Ulrike und Geoffrey Tate (2008). „Superstar CEOs“. Working Paper, University of California, Berkley.

Neal, Alison (Ed.) (2006). The International Who's Who. London: Routledge.

Neckermann, Susanne, Reto Cueni und Bruno S. Frey (2009). „What is an Award Worth? An Econometric Assessment of the Impact of Awards on Employee Performance“. IEW Working Paper No. 411, University of Zurich, Switzerland.

Neckermann, Susanne und Bruno S. Frey (2007). „Awards as Incentives“. IEW Working Paper No. 334, Institute for Empirical Research in Economics, University of Zurich, Switzerland.

Neckermann, Susanne und Michael Kosfeld (2009). „Working for nothing? The Effect of Awards on Employee Performance“. Mimeo, University of Zurich, Switzerland.

Risk, James C. (1972). The History of the Order of the Bath and ist Insignia. London: Spink and Son. 
Rosen, Sherwin (1981). „The Economics of Superstars“. American Economic Review 71(5): 845-858.

Sururov, Anton und Jeroen Van de Ven (2006). „Discretionary rewards as a feedback mechanism“. Mimeo, Amsterdam Center for Law \& Economics, University of Amsterdam.

Young, H. Peyton (1993). „The Evolution of Conventions“. Econometrica 61(1): 5784.

Tab. 1: Durchschnittliche Zahl von Auszeichnungen pro Person nach Land

\begin{tabular}{lclc} 
& $\begin{array}{c}\text { Alle } \\
\text { Auszeichnungen }\end{array}$ & \multicolumn{1}{c}{$\begin{array}{c}\text { Nationale } \\
\text { staatliche } \\
\text { Auszeichnungen }\end{array}$} \\
\hline Durchschnitt & 2.66 & Durchschnitt & 0.43 \\
Varianz & 1.96 & Varianz & 0.11 \\
Oberste 10 Länder in jeweiliger Kategorie: & \\
Kanada & 6.82 & Polen & 1.78 \\
Vereinigtes & & & \\
Königreich & 6.78 & Frankreich & 1.32 \\
Polen & 6.16 & Tunisien & 1.05 \\
Australien & 5.66 & Ägypten & 1.02 \\
Senegal & 5.30 & Malaysien & 1.00 \\
Ungarn & 5.00 & Finnland & 0.88 \\
Neuseeland & 4.96 & Kanada & 0.86 \\
Schweiz & 4.70 & Portugal & 0.84 \\
Finnland & 4.64 & Equador & 0.83 \\
Spanien & 4.20 & Philippinen & 0.82
\end{tabular}

\section{Information zu 7 weiteren Ländern:}

USA

Kanada 
Vereins für Socialpolitik Perspektiven der Wirtschaftspolitik 11(1) (2010): 1-15.

$\begin{array}{lll}\text { Königreich } & & \\ \text { Frankreich } & 3.60 & 1.32 \\ \text { Deutschland } & 2.46 & 0.48 \\ \text { Spanien } & 4.20 & 0.70 \\ \text { Italien } & 1.96 & 0.22\end{array}$


Tab. 2: Auszeichnungen pro Land für in der Wissenschaft tätige Personen
Anteil an allen
Durchschnittliche Zahl von Auszeichnungen Auszeichnungen

\begin{tabular}{|c|c|c|c|}
\hline Durchschnitt & 0.23 & Durchschnitt & 3.76 \\
\hline Varianz & 0.03 & Varianz & 7.62 \\
\hline \multicolumn{4}{|c|}{ Oberste 10 Länder in jeweiliger Kategorie: } \\
\hline Schweiz & 0.66 & Belgien & 10.83 \\
\hline Belgien & 0.66 & Venezuela & 10.00 \\
\hline Türkei & 0.61 & Schweiz & 9.50 \\
\hline Niederlande & 0.56 & Japan & 9.43 \\
\hline Deutschland & 0.55 & USA & 9.43 \\
\hline Australien & 0.53 & Littauen & 9.17 \\
\hline Nigerien & 0.50 & Frankreich & 8.50 \\
\hline Schweden & 0.47 & Polen & 7.67 \\
\hline Israel & 0.45 & Argentinien & 7.60 \\
\hline El Salvador & 0.43 & Australien & 7.50 \\
\hline
\end{tabular}

Information zu 7 weiteren Ländern:

$\begin{array}{lll}\text { USA } & 0.35 & 9.43 \\ \text { Kanada } & 0.21 & 6.00 \\ \text { Vereinigtes } & & 6.50 \\ \text { Königreich } & 0.19 & \\ \text { Frankreich } & 0.28 & 8.50 \\ \text { GDeutschland } & 0.55 & 3.37 \\ \text { Spanien } & 0.06 & 1.71 \\ \text { Italien } & 0.42 & 3.80\end{array}$


Tab. 3: Auszeichnungen in der Wirtschaft

Durschnittliche Zahl von wirtschaftlichen Auszeichnungen pro Person
Anteil der Auszeichnungen in einem Land für wirtschaftliche Aktivitäten

$\begin{array}{llll}\text { Durchschnitt } & 0.06 & \text { Durchschnitt } & 0.02 \\ \text { Varianz } & 0.01 & \text { Varianz } & 0.00\end{array}$

Oberste 10 Länder in jeweiliger Kategorie:

$\begin{array}{llll}\text { Kanada } & 0.52 & \text { Saudi Arabia } & 0.21 \\ \text { Singapur } & 0.46 & \text { Republik Korea, } & 0.15 \\ \text { USA } & 0.34 & \text { Singapur } & 0.15 \\ \text { Saudi Arabien } & 0.27 & \text { Tanzanien } & 0.14 \\ \text { Australien } & 0.26 & \text { USA } & 0.10 \\ \text { Republik Korea } & 0.24 & \text { China } & 0.10 \\ \text { Philippinen } & 0.20 & \text { Israel } & 0.09 \\ \text { Schweden } & 0.18 & \text { Pakistan } & 0.09 \\ \text { Türkei } & 0.16 & \text { Kanada } & 0.08 \\ \text { Israel / Schweiz } & 0.14 & \text { Türkei } & 0.08\end{array}$

Information zu 7 weiteren Ländern:

$\begin{array}{lll}\text { USA } & 0.34 & 0.10 \\ \text { Kanada } & 0.52 & 0.08 \\ \text { Vereinigtes } & & 0.01 \\ \text { Königreich } & 0.04 & \\ \text { Frankreich } & 0.04 & 0.01 \\ \text { Deutschland } & 0.06 & 0.03 \\ \text { Spanien } & 0.06 & 0.02 \\ \text { Italien } & 0.04 & 0.02\end{array}$


Abbildung 1:

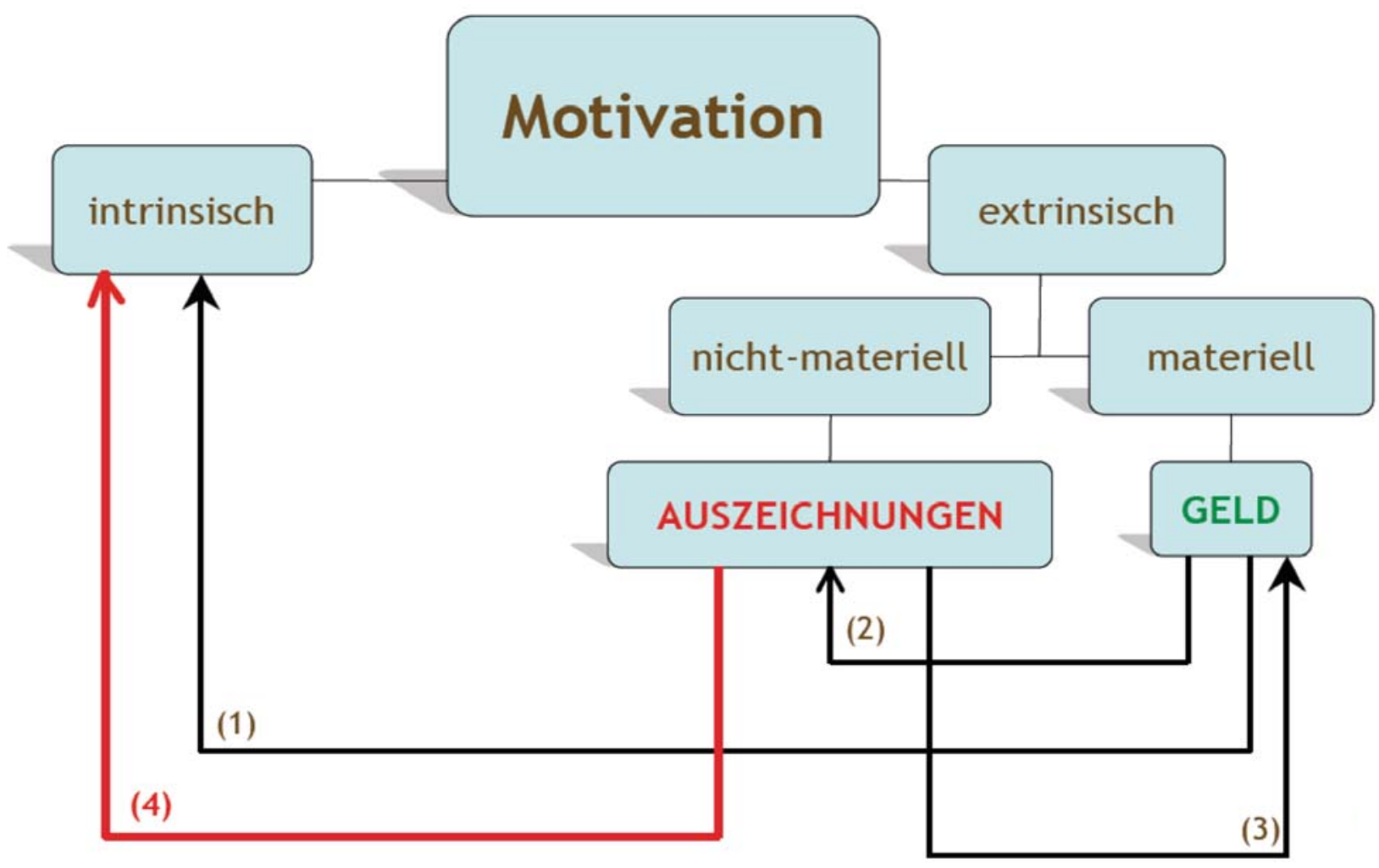

